\title{
Schuldenerlass oder Schuldenerleichterung?
}

\section{Eric Offner}

\section{Relevanz}

Es gibt tausend gute Gründe, wichtige Staatsausgaben mit neuen Schulden zu finanzieren, wenn die Steuern nicht reichen. Reserven aufzubauen und sich auf nachhaltige Finanzpolitik zu verpflichten fällt der Politik dagegen schwer. Ist der Staatshaushalt außer Kontrolle geraten, müssen die Gläubiger entscheiden. Sie können mit einem deutlichen Schuldenschnitt ein Ende mit Schrecken setzen und die Überschuldung kräftig korrigieren, damit wenigsten die restliche Staatsschuld sicher zurückkommt. Wer vorher die Kreditwürdigkeit nicht sorgfältig prüft, muss es eben später nachholen und die Schulden auf ein Niveau reduzieren, das tragbar bleibt. Oder sie einigen sich auf Schuldenerleichterungen und eine Streckung der Rückzahlung, und riskieren damit eine verschleppte Insolvenz anstatt nachhaltiger Gesundung. Ein Schuldenschnitt bietet eher Gewähr, zu neuem Wachstum zurückzufinden und die Tragbarkeit der Staatsschulden wiederherzustellen.

\section{Quelle}

Reinhart, C. M., und C. Trebesch (2016), Sovereign Debt Relief and its Aftermath, Journal of the European Economic Association 14(1), 215-51.

E. Offner $(\square)$

Universität St.Gallen, St.Gallen, Schweiz

E-Mail: eric.athaydeoffner@student.unisg.ch 
Soll hochverschuldeten Staaten ein ein Schuldenerlass gewährt werden? Die Frage ist nicht neu und nach wie vor höchst aktuell. Der Internationale Währungsfonds forderte nachdrücklich einen Schuldenerlass für Griechenland. Der frühere US-Finanzminister Larry Summers schlug einen Schuldenerlass für die kriegszerrüttete Ukraine vor. Dennoch ist über die wirtschaftlichen Folgen eines Schuldenerlasses überraschend wenig bekannt.

Die ökonomische Theorie liefert widersprüchliche Argumente. Einerseits können sowohl Gläubiger als auch Schuldner von einem Teilerlass profitieren, da ein überhöhter Schuldenstand und die Aussicht auf hohe zukünftige Schuldentilgungen inländische Investitionen hemmt. Deshalb sollte nach einem Schuldenerlass das Wachstum zunehmen, was die Rückzahlung der verbleibenden Schulden wahrscheinlicher macht. Andererseits beschädigt ein Zahlungsausfall Reputation und Vertrauen und kann Sanktionen auslösen. Auch mag der Anreiz für mutige Wirtschaftsreformen erlahmen.

Carmen M. Reinhart von der Universität Harvard und Christoph Trebesch von der Universität München gehen der Frage nach, wie sich ein Schuldenerlass auf die wirtschaftliche Entwicklung des betroffenen Landes auswirkt. Sie betrachten zwei unterschiedliche Episoden während des 20. Jahrhunderts.

Zuerst konzentrieren sich die Autoren auf die Zwischenkriegszeit. Sie untersuchen die Verschuldung von 18 entwickelten, überwiegend europäischen Staaten gegenüber den USA und dem Vereinigten Königreich. Die Situation erinnert an die Länder in der Peripherie der Eurozone, wo mittlerweile ebenfalls ein Grossteil der Schulden in den Händen staatlicher Gläubiger liegt. Die Verschuldung der untersuchten Länder bestand nur zum Teil aus Kriegsschulden. Viele Kredite wurden erst nach 1918 aufgenommen etwa für den Wiederaufbau. Die Forscher untersuchen nun zwei Ereignisse, welche alle Schuldner gleichzeitig betrafen: Im Jahr 1931 erliessen die USA mit dem Hoover-Moratorium Zins- und Tilgungszahlungen aller interalliierten Kriegsschulden sowie der deutschen Reparationszahlungen vorübergehend für ein Jahr. Im Sommer 1934 stellten dann 18 der 19 Kreditnehmer die Rückzahlung ihrer Kriegsschulden an die USA und das Vereinigte Königreich trotz Protesten dauerhaft ein.

Zudem analysieren die Forscher die Schuldenerlässe und Umstrukturierungen von 30 Schwellenländern gegenüber privaten Gläubigern (z. B. ausländische Banken) zwischen 1978 und 2010. In den 80er und 90er Jahren koordinierten die Finanzminister der USA, James Baker und Nicolas Brady, zwei Entschuldungsinitiativen, die von den USA und dem IWF unterstützt wurden. Der Baker-Plan 1986 zielte darauf ab, freiwillige Kapitalzuflüsse und Strukturreformen in den Krisenländern zu fördern. Neben zinsvergünstigten Darlehen gehörten dazu eine Reihe von Umschuldungsvereinbarungen, welche die Laufzeit der Schulden um bis zu 15 Jahre verlängerten. Dagegen sah die Brady Initiative 1990 einen Schuldenerlass und damit eine unmittelbare Reduktion der Verschuldung vor. 
Insgesamt wurden 46 Episoden von Schuldenerlässen zusammengefasst, welche die Verschuldung teils erheblich verringerten: Im Durchschnitt betrugen sie zwischen 16 bis $21 \%$ des BIP bzw. zwischen 36 und $43 \%$ der Auslandsverschuldung.

Der durchschnittliche Schuldenerlass betrug in der Zwischenkriegszeit 20.6 Prozent des BIP und bei Schwellenländern (1978-2010) 15.7 Prozent des BIP.

Die Forscher schätzen, wie sich die vier Schuldenerlässe (1931, 1934, 1986, 1990) auf die wirtschaftliche Entwicklung der betroffenen Staaten auswirkten. Dazu betrachten sie das reale Pro-Kopf Einkommen, ein Mass für die Kreditwürdigkeit (Länderkredit-Rating), sowie Schuldenstand und Schuldendienst. Sie vergleichen den Verlauf jener Grössen zwischen Staaten, die von Entschuldungsmassnahmen profitierten, mit jenen, die davon nicht betroffen waren, während jeweils fünf Jahren vor und nach dem Schuldenerlass.

Die Schätzungen für die Zwischenkriegszeit zeigen erhebliche Unterschiede. Das Hoover Moratorium 1931 sah lediglich eine Schuldenerleichterung vor und wirkte sich kaum signifikant auf die wirtschaftliche Entwicklung der betroffenen Staaten aus. Nach dem Schuldenerlass in Folge der Zahlungsausfälle von 18 Staaten im Sommer 1934 stieg hingegen das reale Wirtschaftswachstum um 4.7 Prozentpunkte pro Jahr. Nach fünf Jahren war das BIP dadurch um rund $20 \%$ höher. Schuldendienst und Verschuldungsquote gingen tendenziell zurück, während sich die Kreditwürdigkeit gegenüber der Vergleichsgruppe verbesserte. Insgesamt deuten die Ergebnisse darauf hin, dass nur eine Schuldentilgung wie 1934 Wachstum und Nachhaltigkeit der verbleibenden Staatsschulden verbessern konnte. Ein vorübergehendes Moratorium, wodurch die Schuldenlast langfristig bestehen blieb, veränderte hingegen die wirtschaftliche Situation kaum.

Nach dem Zahlungsausfall 1934 mit anschliessendem Schuldenerlass nahm das reale Pro-Kopf-Wachstum in den betroffenen 18 Staaten um 4.7 Prozentpunkte pro Jahr zu.

Bei den Schwellenländern erkennen die Forscher ähnliche Trends, wie Abb. 1 illustriert. Sie zeigt die durchschnittliche Entwicklung des realen BIP von Staaten, die Hilfe erhalten haben, und von einer Vergleichsgruppe vor und nach den jeweiligen Initiativen. Das linke Diagramm deutet darauf hin, dass die Schuldenerleichterungen des Baker Plans 1986 das Wachstum in den teils hoch verschuldeten Krisenländern nicht erhöhen konnten. Die Staatsschuldenquote nahm sogar weiter zu, obwohl sich die Bonität verbesserte und der Schuldendienst zurückging. Zwar führte der Baker Plan zu einer Entlastung des Budgets, 

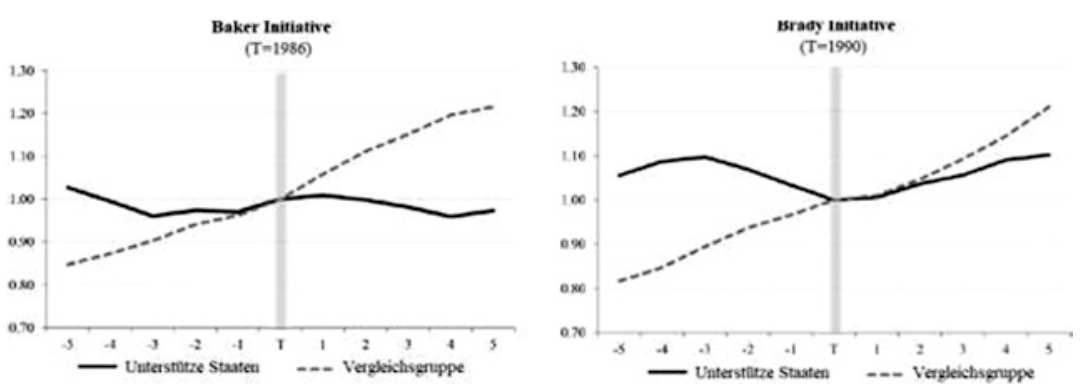

Abb. 1 Entwicklung des realen Pro-Kopfeinkommens jeweils 5 Jahre vor nach einer Schuldenerleichterung. (Quelle: Reinhart und Trebesch 2016, Abb. 9)

brachte aber kaum darüber hinausführende Verbesserungen mit sich. Dagegen macht das rechte Diagramm die Wirksamkeit der Brady Initiative 1990 deutlich, welche mit einem Schuldenerlass in den folgenden fünf Jahren mit einer um drei Prozentpunkte höheren Wachstumsrate einherging. Die Kreditwürdigkeit gemessen am Länderkredit-Rating verbesserte sich um $21 \%$ nach zwei bzw. $40 \%$ nach fünf Jahren.

Die Brady-Initiative 1990 sah Schuldenerlässe vor und steigerte in den betroffenen Staaten die reale Wachstumsrate um 3 Prozentpunkte pro Jahr. Die Kreditwürdigkeit verbesserte sich nach fünf Jahren um 40 Prozent.

Eine reine Umschuldung und Streckung der Zinslasten tragen wenig zur wirtschaftlichen Erholung überschuldeter Länder bei. Das Wachstum nimmt meist erst nach einem grossen Schuldenerlass wieder zu, wie z. B. in Folge der Brady-Initiative. Jedoch legen diese Ergebnisse keinesfalls den Schluss nahe, dass die Folgen anderer Formen von Schulderleichterungen quantitativ unwirksam gewesen wären. Fiskalische Einschnitte, Strukturreformen, finanzielle Repression und Inflation fanden in den betrachteten Fällen häufig gleichzeitig statt und haben oft wesentlich zur Beseitigung eines hohen Schuldenüberhangs beigetragen. 
Open Access Dieses Kapitel wird unter der Creative Commons Namensnennung 4.0 International Lizenz (http://creativecommons.org/licenses/by/4.0/deed.de) veröffentlicht, welche die Nutzung, Vervielfältigung, Bearbeitung, Verbreitung und Wiedergabe in jeglichem Medium und Format erlaubt, sofern Sie den/die ursprünglichen Autor(en) und die Quelle ordnungsgemäß nennen, einen Link zur Creative Commons Lizenz beifügen und angeben, ob Änderungen vorgenommen wurden.

Die in diesem Kapitel enthaltenen Bilder und sonstiges Drittmaterial unterliegen ebenfalls der genannten Creative Commons Lizenz, sofern sich aus der Abbildungslegende nichts anderes ergibt. Sofern das betreffende Material nicht unter der genannten Creative Commons Lizenz steht und die betreffende Handlung nicht nach gesetzlichen Vorschriften erlaubt ist, ist für die oben aufgeführten Weiterverwendungen des Materials die Einwilligung des jeweiligen Rechteinhabers einzuholen.

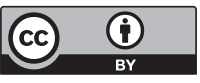

